\title{
Transfermoral aortic valve implantation using self-expanding New Valve Technology (NVT) Allegra bioprosthesis: A pilot prospective study
}

\author{
Dariusz Jagielak ${ }^{1}$, Aleksandra Stanska ${ }^{1}$, Andrzej Klapkowski ${ }^{1}$, Maciej Brzezinski ${ }^{1}$, \\ Maciej Kowalik ${ }^{1}$, Dariusz Ciecwierz ${ }^{2}$, Milosz Jaguszewski ${ }^{2}$, Marcin Fijalkowski ${ }^{2}$ \\ ${ }^{1}$ Department of Cardiac and Vascular Surgery, Medical University of Gdansk, Poland \\ ${ }^{2} 1^{\text {st }}$ Department of Cardiology, Medical University of Gdansk, Poland
}

This paper was guest edited by Prof. Wojciech Wojakowski

\begin{abstract}
Background: Transcatheter aortic valve implantation (TAVI) has become a standard therapeutic option for patients with severe aortic stenosis (AS) at high cardiac surgical risk. The aim of the NAUTILUS study was to investigate the safety and performance of the New Valve Technology (NVT) Allegra bioprosthesis in high-risk patients undergoing TAVI.

Methods: Twenty seven patients with severe, symptomatic AS at high surgical risk were prospectively enrolled, who underwent treatment using the novel self-expanding NVT Allegra bioprosthesis via transfemoral approach (TF-TAVI). The primary end-point was all-cause mortality at 30 days.

Results: Patients were elderly (83 years, range 75-89 years), and predominantly female (70.4\%, $n=19$ ). All patients were deemed to be at high surgical risk, with a mean logistic EuroSCORE of $12.4 \%$ (range, $2.8-31.8 \%)$. The bioprosthesis was successfully implanted in $96 \%$ of the cases $(n=25)$. The echocardiographic assessment confirmed good hemodynamic profile after implantation of the NVT Allegra bioprosthesis. Complications included cardiac tamponade $(4 \%, n=1)$ and the need for permanent pacemaker implantation $(8 \%, n=2)$. The analysis of procedural aspects showed a short learning effect related to the precise placement of the valve. A significant improvement in clinical symptoms were observed, and no patients died in-hospital or within 30 days of post-discharge observation.

Conclusions: This prospective observation shows that the NVT Allegra bioprosthesis was associated with a satisfactory safety profile and a remarkable hemodynamic performance after implantation. (Cardiol J 2021; 28, 3: 384-390)

Key words: aortic stenosis, clinical trial, elderly population, transcatheter aortic valve implantation
\end{abstract}

\section{Introduction}

Transcatheter aortic valve implantation (TAVI) has evolved from a challenging and hazardous procedure to a minimally invasive, safe, and predictable treatment strategy. It has become a standard treatment for intermediate and high-risk patients with severe aortic stenosis (AS) [1]. Since 2002, when the first TAVI was performed [2], a large number of studies have proven its clinical utility and low rates of procedure-related complications [3]. Currently, in some countries, the annual rate of TAVIs surpassed the surgical replacement procedure [4]. Even though there are many Conformité Européenne (CE) marked devices available, there is still a strong need for further technological improvement and new valve design. In spite of the procedural success, undesirable, adverse events related to the TAVI procedure or TAVI system are still reported in up to $4 \%$ of cases [5-10]. Im-

Address for correspondence: Dariusz Jagielak, MD, PhD, Department of Cardiac and Vascular Surgery, ul. Dębinki 7, 80-241 Gdańsk, Poland, tel: +48 509487577, e-mail: darjag@gumed.edu.pl

Received: 25.07.2018 Accepted: 23.01.2019

This article is available in open access under Creative Common Attribution-Non-Commercial-No Derivatives 4.0 International (CC BY-NC-ND 4.0) license, allowing to download articles and share them with others as long as they credit the authors and the publisher, but without permission to change them in any way or use them commercially. 


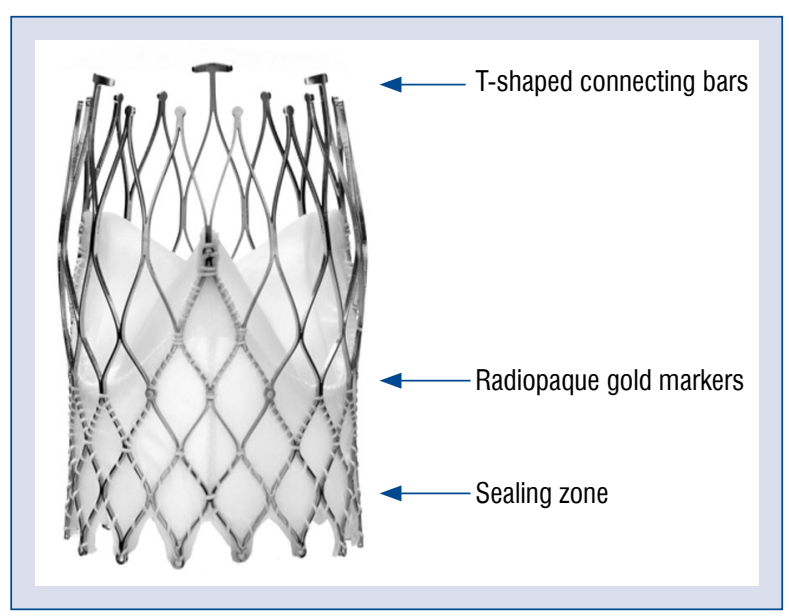

Figure 1. Design of the Allegra valve.

provement of technology may have the potential to minimize the complication rate [11].

The New Valve Technology (NVT) Allegra system is a novel self-expanding TAVI device designed to overcome some limitations of the first-generation systems, including malpositioning, paravalvular leakage (PVL), hemodynamic compromise, and necessity for pacemaker implantation. The valve is implanted in a three-step deployment process that ensures accurate final positioning (Fig. 1). The first-in-human experience showed a high rate of procedural success, satisfactory hemodynamic results, and clinical symptom improvement [12].

The purpose of this study was to assess the safety and performance of the novel NVT Allegra self-expanding aortic prosthesis using a transfemoral approach. The study provides 30 -day outcomes in a high-risk patient setting, treated in a wellexperienced TAVI center.

\section{Methods}

\section{Study overview and patient population}

NAUTILUS (NVT trAnsfemoral mUlticentric aorTIc valve pivotaL stUdy for Safety and effectiveness) was a single-arm clinical study conducted at 8 centers in 3 countries (Switzerland, Poland, and Brazil), designed to assess the safety and performance of the NVT Allegra TAVI System (NVT, $\mathrm{GmbH}$, Hechingen, Germany).

Herein, is presented the largest single-center experience with the novel NVT Allegra system implanted in 26 patients accepted by the Heart Team to undergo transfemoral transcatheter aortic valve implantation (TF-TAVI). All patients suffered from severe, symptomatic AS and met the
NAUTILUS eligibility criteria. The main inclusion criteria were: 1 ) age $\geq 75$ years; 2 ) symptomatic (New York Heart Association [NYHA] class II or greater) severe degenerative native AS (mean transvalvular pressure gradient $>40 \mathrm{mmHg}$ and/ /or aortic jet velocity $>4.0 \mathrm{~m} / \mathrm{s}$ and/or aortic valve area of $<1.0 \mathrm{~cm}^{2}$ [or aortic valve area index $\left.\leq 0.6 \mathrm{~cm}^{2} / \mathrm{m}^{2}\right]$ ); 3) high risk for surgical aortic valve replacement with a logistic EuroSCORE $\geq 20 \%$, or documented agreement of the Heart Team that the patient is at high risk for surgery due to frailty and/ /or coexisting comorbidities.

Amongst others, the protocol defined exclusion criteria comprised: 1) unicuspid or bicuspid valve disease; 2 ) non-calcified aortic valve disease; 3) mixed valve disease with predominant aortic regurgitation greater than $3+$ or with associated severe (greater than $3+$ ) mitral regurgitation; 4) aortic annulus size $<19 \mathrm{~mm}$ or $>29 \mathrm{~mm}$; 5) type of femoral access, or any other anatomical conditions that prevented safe placement of an 18 French introducer sheath and manipulation of the TAVI system (e.g. severe femoral-iliac obstructive calcification or tortuosity) [13].

All patients were followed-up for 30 days. Written informed consent was obtained from each participant. The study was approved by the local ethics committee and the competent authority.

\section{NVT Allegra system and the TAVI implantation protocol}

The NVT Allegra transcatheter heart valve (Fig. 1) is a self-expanding device, designed to avoid hemodynamic compromise and to facilitate correct positioning during the subsequent steps of implantation. The valve consists of a nitinol stent frame and bovine pericardium (annular skirt and leaflets). The stent frame has a closed cell, diamond-shaped configuration. The variable cell size distribution allows for better coronary perfusion and easier access for any further percutaneous coronary interventions. Six radiopaque gold markers are incorporated into the stent frame. They indicate the distal part of the semilunar valve to facilitate correct valve positioning. The $12-\mathrm{mm}$ bovine pericardial sealing skirt reduces the risk of significant PVL. The NVT Allegra valve is available in 3 sizes $(23,27$ and $31 \mathrm{~mm})$, with a frame height of $37.3,41.3$, and $43.0 \mathrm{~mm}$, respectively, (Table 1 ) covering aortic annulus diameter sizes from 19 to $29 \mathrm{~mm}$.

The delivery system has an 18 French cartridge and a 15 French catheter shaft. The grip uses a Squeeze-to-Release ${ }^{\circledR}$ mechanism, which prevents rotation during a stepwisely performed implanta- 
Table 1. Specifications of New Valve Technology (NVT) Allegra transcatheter valve.

\begin{tabular}{lccc}
\hline & NVT 23 & NVT 27 & NVT 31 \\
\hline Frame height [mm] & 37.3 & 41.3 & 43.0 \\
Inflow diameter [mm] & 23.8 & 27.4 & 31.0 \\
Outflow diameter [mm] & 20.8 & 24.0 & 24.0 \\
$\begin{array}{l}\text { Tissue annulus } \\
\text { diameter [mm] }\end{array}$ & $19-22$ & $22-25$ & $25-29$ \\
\hline
\end{tabular}

tion. The prosthesis is released in a three-step deployment mechanism. The novel implantation technology (PermaFlow ${ }^{\circledR}$; New Valve Technology) facilitates positioning of the Allegra valve and prevents interference with the left ventricular outflow tract. In the first step of the implantation, the middle part of the valve is released, while both ends of the device are still captured (Fig. 2). During the second step, the bottom of the valve is released (Fig. 3). The operator can assess valve position, patency of the coronaries, hemodynamic functions, and paravalvular regurgitation. The process can be re-started till this step, and the valve can thus be retrieved. In the third step, when the operator confirms the position of the prosthesis, the safety locker is released for final deployment of the NVT Allegra valve.

All procedures were performed via the open transfemoral approach for TAVI (TF-TAVI) under local or general anaesthesia. According to the protocol, pre-dilatation before valve implantation was mandatory.

\section{Data management and statistical analysis}

The data for the clinical trial was collected prospectively during hospitalization (baseline and discharge) and after 30 days in an outpatient setting by using a dedicated electronic case report form. Each patient underwent transthoracic echocardiography assessment at least three times - before the implantation, after the procedure and/or at discharge and after 1 month. Hemodynamic prosthesis performance assessment estimation included maximal velocity, mean pressure gradient, effective orifice area, and presence and grade of PVL. Results were presented as numbers of patients (percentages) or means, where applicable. Statistical analysis was performed using STATISTICA 12.0 PL (StatSoft, Krakow, Poland).

\section{Results}

Twenty-seven patients were considered eligible for the treatment and signed the informed

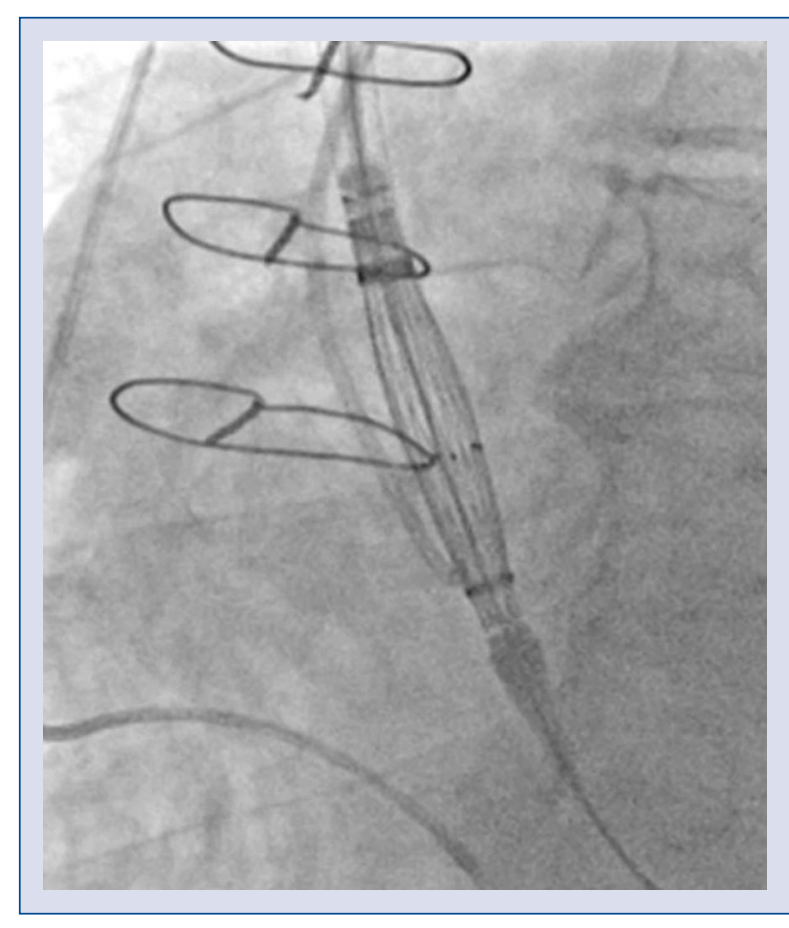

Figure 2. The Allegra valve positioning.

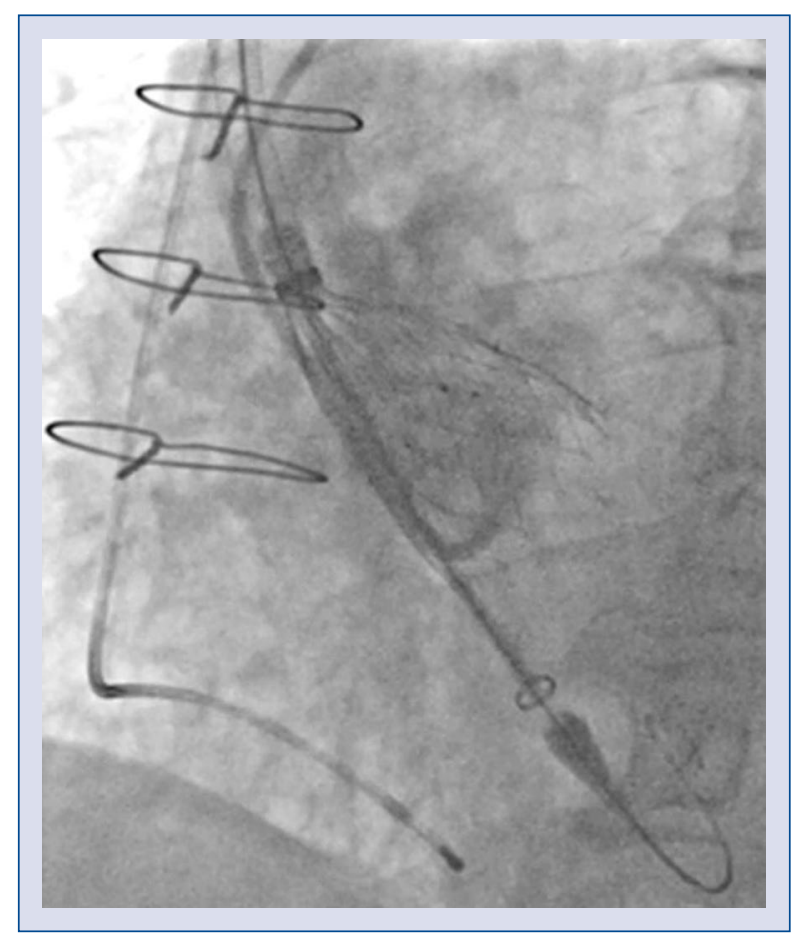

Figure 3. Releasing of the bottom of the valve.

consent. One patient underwent isolated balloon valvuloplasty of the native aortic valve due to unexpected anatomical conditions; massive, bulky asymmetric calcification. Therefore, 26 patients 
underwent attempted implantation of the NVT Allegra valve.

The patient population was elderly with a mean age of 83 years (range, $75-89$ years), $70.4 \%$ were female, and the mean logistic EuroSCORE was $12.4 \%$ (range, $2.8-31.8 \%$ ). Patient characteristics and parameters at baseline are presented in Table 2.

The implantation of a single functioning NVT bioprosthesis in the correct annular position was successfully completed in 25 patients. Due to the dislocation of the prosthesis into the left ventricle, 1 patient required open-heart aortic valve replacement. Flow chart of the study is presented (Fig. 4). The dislocation of the valve was caused by the combination of a relatively low degree of calcification of the annulus, and disconnection of the prosthesis from the delivery system.

Most events were considered as expected and documented in the case report form (Table 3). Two patients could not attend the clinic for a follow-up visit at 30 days and clinical data were collected by the phone. No patient died within the period of 30 days (Table 4 ).

The echocardiographic assessment immediately after valve implantation and before discharge showed excellent hemodynamic performance, which was maintained at 30 days (Table 5). At 30 days, the majority $(84 \%)$ had only mild or less residual aortic regurgitation, while $16 \%$ moderate and $0 \%$ severe aortic regurgitation was noted (Table 6).

\section{Discussion}

This is the largest single-center study of the novel transcatheter self-expanding NVT Allegra bioprosthesis transfemoral system. The main purpose of the NAUTILUS study was to assess safety and performance of the NVT Allegra valve. The new valve had a favorable post-deployment hemodynamic profile, with low post-procedural transvalvular gradients. No cardiac death or cerebrovascular incidents, as well as a very low permanent pacemaker implantation rate was recorded at 30 days. These results suggest that the novel valve has a good safety and efficacy profile and could be used in patients with severe symptomatic AS.

Given the dynamic development of the procedure and different product refinements, the field of transcatheter heart valve systems is currently highly competitive, with a wide spectrum of various CE-marked devices. However, TAVI is still associated with a risk of severe complications,
Table 2. Preoperative characteristics and parameters $(n=27)$.

\begin{tabular}{lc}
\hline Age [years] & $83(75-89)$ \\
Gender: & \\
Male & $8(29.6 \%)$ \\
$\quad$ Female & $19(70.4 \%)$ \\
Logistic EuroSCORE [\%] & $12.4(2.8-31.8)$ \\
Hypertension & $22(81.5 \%)$ \\
Diabetes mellitus & $14(51.8 \%)$ \\
COPD & $5(18.5 \%)$ \\
Coronary artery stenosis (>50\%) & $2(7.4 \%)$ \\
Previous myocardial infarction & $7(25.9 \%)$ \\
Previous coronary surgery & $4(14.8 \%)$ \\
Previous coronary angioplasty & $12(44.4 \%)$ \\
Previous stroke or TIA & $1(3.7 \%)$ \\
Creatinine clearance (<6 mL/min) & $7(25.9 \%)$ \\
NYHA: & \\
I & \\
II & - \\
III & $3(11.1 \%)$ \\
IV & $23(85.2 \%)$ \\
Conduction disorders & $1(3.7 \%)$ \\
(LBBB, RBBB, AVB) & $2(7.4 \%)$ \\
Pre-existing permanent pacemaker & $3(11.1 \%)$ \\
Aortic valve insufficiency ( $\geq$ mild) & $11(40.7 \%)$ \\
Mitral valve insufficiency ( $\geq$ mild) & $18(66.7 \%)$ \\
\hline AVB & \\
\hline
\end{tabular}

AVB - atrio-ventricular block; COPD - chronic obstructive pulmonary disease; LBBB - left bundle branch block; NYHA — New York Heart Association functional class; RBBB — right bundle branch block; TIA — transient ischemic attack

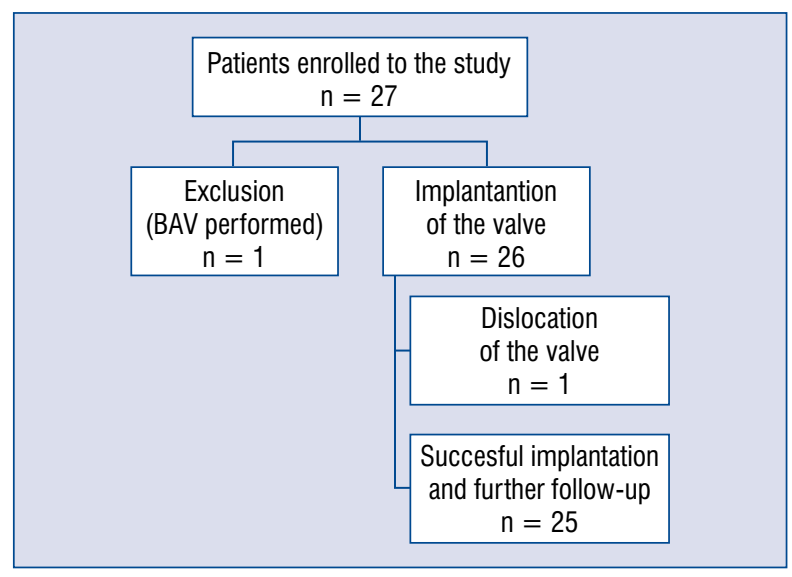

Figure 4. Flow chart of the study; BAV — balloon aortic valvuloplasty.

which may occur in up to $5 \%$ of procedures [1]. The contemporary TAVI system design should provide easy positioning, and an option to retrieve 
Table 3. Procedural characteristics $(n=27)$.

$\begin{array}{lc}\text { Procedural success } & 25(92.6 \%) \\ \text { (one prosthesis implanted) } & \\ \text { Failed study valve implantation: } & 2(7.4 \%) \\ \text { Valve-in-valve } & 0(0 \%) \\ \text { Conversion to other TAVI } & 0(0 \%) \\ \text { Conversion to surgical AVR } & 1(3.7 \%) \\ \text { Unexpected anatomical conditions } & 1(3.7 \%) \\ \text { Attempted valve retrieval: } & \\ \text { Successful retrieval } & 2(7.4 \%) \\ \text { Failed retrieval } & 0(0 \%) \\ \text { Fluoroscopy time [min] } & 17.6(7-60) \\ \text { Volume of contrast [mL] } & 107(60-290) \\ \text { Valve size ( } \mathrm{n}=27): & \\ 23 \mathrm{~mm} & 0(0 \%) \\ 27 \mathrm{~mm} & 15(55.6 \%) \\ 31 \mathrm{~mm} & 11(40.7 \%) \\ \text { Need for post-dilatation } & 0(0 \%)\end{array}$

AVR - aortic valve replacement; TAVI — transcatheter aortic valve implantation

Table 4. Prevalence of clinical outcomes and adverse events $(n=25)$.

\begin{tabular}{ll} 
All-cause mortality & $0(0 \%)$ \\
$\begin{array}{l}\text { Permanent pacemaker implantation } \\
\text { due to atrio-ventricular block }\end{array}$ & $2(8 \%)$ \\
Sepsis & $1(4 \%)$ \\
Any stroke & $0(0 \%)$ \\
Pericardium drainage due to late & $1(4 \%)$ \\
cardiac tamponade & \\
Renal failure & $0(0 \%)$ \\
Respiratory failure & $1(4 \%)$ \\
Major vascular complication & $0(0 \%)$ \\
Minor vascular complication & $0(0 \%)$ \\
Acute myocardial infarction & $0(0 \%)$ \\
Minor bleeding (sheath failure) & $1(4 \%)$ \\
\hline
\end{tabular}

the device when the position of the valve is compromised. A reduced rate of complications related
Table 6. The prevalence of paravalvular leakage (PVL) on discharge day and 30-days follow-up (FU).

\begin{tabular}{lcc}
\hline PVL & $\begin{array}{c}\text { Discharge } \\
(\mathbf{n}=25)\end{array}$ & $\begin{array}{c}30 \text {-days FU } \\
(\mathbf{n}=23)\end{array}$ \\
\hline None & $3(12 \%)$ & $3(13 \%)$ \\
Mild & $18(72 \%)$ & $16(69.6 \%)$ \\
Moderate & $4(16 \%)$ & $4(17,4 \%)$ \\
Severe & $0(0 \%)$ & $0(0 \%)$ \\
\hline
\end{tabular}

to the malposition of the prosthesis such as PVL, pacemaker implantation or overlie of coronary ostia is essential for improving long-term results.

In this study, a high rate of procedural success was demonstrated with the Allegra NVT bioprosthesis $(96 \%)$. One of the greatest advantages of this new system is the ability to reposition and retrieve the device in cases of malposition or a suboptimal result. In the present cohort, the device had to be retrieved in 2 patients. The retrieving maneuvers and second deployment were easily controlled without complications. The Allegra system has features allowing for very precise positioning. The presence of radiopaque markers in the delivery system as well as at the transition between the annular skirt and the bottom of the leaflets, markedly facilitates the procedure by enabling direct and clear visualization of the optimal implantation height and limits of the sealing skirt.

The overall risk of permanent atrio-ventricular conduction disturbances following TAVI procedures varies, but remains around 17\% [14]. In comparison to balloon expandable valves, the selfexpandable (nitinol-based) TAVI valve prostheses have a slightly higher rate of postprocedural atrioventricular conduction block, requiring pacemaker implantation $(28 \%)[15,16]$. In this study, only $8 \%$ of patients required pacemaker implantation, and incomparison to other self-expandable valve prostheses, this is a very promising result. This may be related to a high valve implantation facilitated by refined deployment technology and valve design.

Table 5. Echocardiographic aortic valve function from baseline until 30 days of patients successfully treated with the study prosthesis.

\begin{tabular}{lccc}
\hline & Baseline $(\mathbf{n}=\mathbf{2 7})$ & Discharge $(\mathbf{n}=\mathbf{2 5})$ & 30-days $(\mathbf{n}=\mathbf{2 3})$ \\
\hline Peak gradient $[\mathrm{mmHg}]$ & $87(58-138)$ & $16.9(8-42)$ & $15.9(8-27)$ \\
Mean gradient $[\mathrm{mmHg}]$ & $56.8(35-91)$ & $9.4(4-21)$ & $9(4-16)$ \\
Peak aortic velocity $[\mathrm{m} / \mathrm{s}]$ & $4.6(4-6)$ & $2(1-3)$ & $2(1-3)$ \\
Effective orifice area $\left[\mathrm{cm}^{2}\right]$ & $0.63(0-1)$ & $1.4(1-2)$ & $1.5(1-2)$ \\
\hline
\end{tabular}


The releasing mechanism of the Allegra allows for a stable and stepwise delivery of the valve, which starts to function early in the deployment process, preventing hemodynamic collapse.

Despite the supra-annular design, the Allegra valve has a reduced frame height to facilitate manipulation and deployment. Short TAVI systems with supra-annular construction are advantageous for the percutaneous treatment of degenerated surgical bioprostheses $[11,12]$. However, patients with non-native aortic valve stenosis were excluded from the study.

The durability of transcatheter aortic valve bioprostheses still remains unknown. The first TAVI procedures were performed in the early 2000 s, and the age and clinical profile of that early group of patients make a clear evaluation of TAVI durability very difficult. Patients with either serious co-morbidities and/or are elderly have restricted prediction of life expectancy. Transferring TAVI to lower-risk, younger patients with longer life expectancy increases the requirements regarding freedom from structural valve deterioration. Randomized trials in this group of patients are already under way [13]. The novel Allegra system tested in the present study incorporates a feature that aims to improve a long-term bioprosthesis functional life. The upper portion of its nitinol frame, to which the commissures of the valve leaflets are attached, has increased compliance and is able to partially flex, accommodating the forces generated across the cardiac cycle, reducing the mechanical stress on the leaflets. Reduction of the stress on the biological part of the valve (i.e. pericardial leaflets) would result in enhanced valve durability. Obviously, such an effect cannot be evaluated in the 30-day results presented in the current study and requires longer observation periods.

Moderate-aortic regurgitation occurred in $16 \%$ of patients at 30 days, which is proportional to other self-expandable-valve-studies [4]. Moreover, it is noteworthy that there was no severe PVL in the present setting. Altogether, it is not possible to rule out whether these events are inherent to valve design or complications that can be minimized as experience with the prosthesis grows and the learning curve levels out. Nevertheless, the results presented are promising, particularly when compared to other TAVI devices in similar clinical scenarios.

Stroke and other cerebrovascular events (CVE) remain common complications following TAVI, ranging between $1.5 \%$ and $4 \%$ [17-19]. The incidence of CVEs is highly related to a higher mortality rate and longer hospital stay [19, 20]. In the current subset, not a single procedure was complicated with cerebrovascular incidents in the 30-day follow-up period. Compared to other studies, this is an outstanding result, confirming safety of the procedure and prosthesis itself. It requires further observation, nevertheless, the first month is usually the most vulnerable period for CVE incidence.

Surgical cut-down was used as vascular access in all cases. There were no vascular complications. The valve has good transition and controllability, but estimation of iliofemoral anatomy is crucial in prevention of vascular events.

The Allegra system is intuitive and easy to use. The learning curve regarding the precision of placement of the device is short.

\section{Limitations of the study}

The present study has some important shortcomings. It is a single-arm study of relatively small sample size, which per se precludes any in-depth comparison against a control group or detailed analyses related to uncommonly-occurring events. Furthermore, the evaluation was focused on the short-term safety and effectiveness, and no inferences can be drawn regarding long-term outcomes after implantation of the device.

\section{Conclusions}

This prospective study shows a satisfactory safety and performance profile of NVT Allegra bioprosthesis. This novel valve prosthesis has an excellent hemodynamic performance and low rate of pacemaker implantations. The analysis of procedural aspects showed a favorable learning curve related to precision of placement of the prosthesis.

\section{References}

1. Aldalati O, MacCarthy P, Dworakowski R. Trans-catheter aortic valve implantation: Contemporary practice and the future. Cardiol J. 2017; 24(2): 206-215, doi: 10.5603/CJ.a2017.0022, indexed in Pubmed: 28248406.

2. Cribier A, Eltchaninoff H, Bash A, et al. Percutaneous transcatheter implantation of an aortic valve prosthesis for calcific aortic stenosis: first human case description. Circulation. 2002; 106(24): 3006-3008, indexed in Pubmed: 12473543.

3. Giordana F, D’Ascenzo F, Nijhoff F, et al. Meta-analysis of predictors of all-cause mortality after transcatheter aortic valve implantation. Am J Cardiol. 2014; 114(9): 1447-1455, doi: 10.1016/j. amjcard.2014.07.081, indexed in Pubmed: 25217456.

4. Eggebrecht H, Mehta RH. Transcatheter aortic valve implantation (TAVI) in Germany 2008-2014: on its way to standard therapy for aortic valve stenosis in the elderly? EuroIntervention. 2016; 11(9): 1029-1033, doi: 10.4244/EIJY15M09_11, indexed in Pubmed: 26384006. 
5. Urena M, Webb JG, Eltchaninoff $\mathrm{H}$, et al. Late cardiac death in patients undergoing transcatheter aortic valve replacement: incidence and predictors of advanced heart failure and sudden cardiac death. J Am Coll Cardiol. 2015; 65(5): 437-448, doi: 10.1016/j. jacc.2014.11.027, indexed in Pubmed: 25660921.

6. Ribeiro HB, Nombela-Franco L, Muñoz-García AJ, et al. Predictors and impact of myocardial injury after transcatheter aortic valve replacement: a multicenter registry. J Am Coll Cardiol. 2015; 66(19): 2075-2088, doi: 10.1016/j.jacc.2015.08.881, indexed in Pubmed: 26541917.

7. Gensas CS, Caixeta A, Siqueira D, et al. Predictors of permanent pacemaker requirement after transcatheter aortic valve implantation: insights from a Brazilian registry. Int J Cardiol. 2014; 175(2): 248-252, doi: 10.1016/j.ijcard.2014.05.020, indexed in Pubmed: 24880480.

8. Wenaweser P, Stortecky S, Heg D, et al. Short-term clinical outcomes among patients undergoing transcatheter aortic valve implantation in Switzerland: the Swiss TAVI registry. EuroIntervention. 2014; 10(8): 982-989, doi: 10.4244/EIJV10I8A166, indexed in Pubmed: 24694729.

9. Ribeiro HB, Webb JG, Makkar RR, et al. Predictive factors, management, and clinical outcomes of coronary obstruction following transcatheter aortic valve implantation: insights from a large multicenter registry. J Am Coll Cardiol. 2013; 62(17): 1552-1562, doi: 10.1016/j.jacc.2013.07.040, indexed in Pubmed: 23954337.

10. Athappan G, Patvardhan E, Tuzcu EM, et al. Incidence, predictors, and outcomes of aortic regurgitation after transcatheter aortic valve replacement: meta-analysis and systematic review of literature. J Am Coll Cardiol. 2013; 61(15): 1585-1595, doi: 10.1016/j.jacc.2013.01.047, indexed in Pubmed: 23500308.

11. Tchetche D, Van Mieghem NM. New-generation TAVI devices: description and specifications. EuroIntervention. 2014; 10 Suppl U: U90-U9U100, doi: 10.4244/EIJV10SUA13, indexed in Pubmed: 25256338.

12. Wenaweser P, Stortecky S, Schütz T, et al. Transcatheter aortic valve implantation with the NVT Allegra transcatheter heart valve system: first-in-human experience with a novel self-expanding transcatheter heart valve. EuroIntervention. 2016; 12(1): 71-77, doi: 10.4244/EIJV12I1A13, indexed in Pubmed: 27173865.

13. Leon MB, Piazza N, Nikolsky E, et al. Standardized endpoint definitions for Transcatheter Aortic Valve Implantation clinical tri- als: a consensus report from the Valve Academic Research Consortium. J Am Coll Cardiol. 2011; 57(3): 253-269, doi: 10.1016/j. jacc.2010.12.005, indexed in Pubmed: 21216553.

14. Siontis GCM, Jüni P, Pilgrim T, et al. Predictors of permanent pacemaker implantation in patients with severe aortic stenosis undergoing TAVR: a meta-analysis. J Am Coll Cardiol. 2014; 64(2): 129-140, doi: 10.1016/j.jacc.2014.04.033, indexed in Pubmed: 25011716.

15. Hayashida K, Morice MC, Chevalier B, et al. Sex-related differences in clinical presentation and outcome of transcatheter aortic valve implantation for severe aortic stenosis. J Am Coll Cardiol. 2012; 59(6): 566-571, doi: 10.1016/j.jacc.2011.10.877, indexed in Pubmed: 22300690.

16. Khawaja MZ, Rajani R, Cook A, et al. Permanent pacemaker insertion after CoreValve transcatheter aortic valve implantation: incidence and contributing factors (the UK CoreValve Collaborative). Circulation. 2011; 123(9): 951-960, doi: 10.1161/CIRCULATIONAHA.109.927152, indexed in Pubmed: 21339482.

17. Eggebrecht H, Schmermund A, Voigtländer T, et al. Risk of stroke after transcatheter aortic valve implantation (TAVI): a meta-analysis of 10,037 published patients. EuroIntervention. 2012; 8(1): 129-138, doi: 10.4244/EIJV8I1A20, indexed in Pubmed: 22391581.

18. Chieffo A, Buchanan GL, Van Mieghem NM, et al. Transcatheter aortic valve implantation with the Edwards SAPIEN versus the Medtronic CoreValve Revalving system devices: a multicenter collaborative study: the PRAGMATIC Plus Initiative (PooledRotterdAm-Milano-Toulouse In Collaboration). J Am Coll Cardiol. 2013; 61(8): 830-836, doi: 10.1016/j.jacc.2012.11.050, indexed in Pubmed: 23333140.

19. Tchetche D, Farah B, Misuraca L, et al. Cerebrovascular events post-transcatheter aortic valve replacement in a large cohort of patients: a FRANCE-2 registry substudy. JACC Cardiovasc Interv. 2014; 7(10): 1138-1145, doi: 10.1016/j.jcin.2014.04.018, indexed in Pubmed: 25240554.

20. Stortecky S, Windecker S, Pilgrim T, et al. Cerebrovascular accidents complicating transcatheter aortic valve implantation frequency, timing and impact on outcomes. EuroIntervention. 2012; 8(1): 62-70, doi: 10.4244/EIJV8I1A11, indexed in Pubmed: 22580250 . 